\title{
Efficacy of Dialectical Behavior Therapy on Clinical Signs and Emotion Regulation in Patients with Obsessive-Compulsive Disorder
}

\author{
Masood Ahovan 1 \\ Saeede Balali2 \\ Najmeh Abedi Shargh ${ }^{*}$ \\ Younes Doostian 4 \\ 1 Departmant of Counseling, Faculty of Psychology and Education, University of Allameh Tabatabai, Tehran, Iran \\ 2 Department of Psychology, Neyshabur Branch, Islamic Azad University, Neyshabur, Iran \\ ${ }^{3}$ Health Promotion Research Center, Zahedan University of Medical Sciences, Zahedan, Iran \\ ${ }^{*}$ Correspondence: Najmeh Abedi Shargh, Health Promotion Research Center, Zahedan University of Medical Sciences, Daneshgah \\ street, Zahedan 987-98138, Iran. Tel: 98-54-332-95745. E-mail: Najme.Abedi@gmail.com \\ ${ }^{4}$ Ph.D student of Rehabilitation Counseling, University of Social Welfare and Rehabilitation Sciences - Tehran - Iran
}

Doi:10.5901/mjss.2016.v7n4p

Abstract

Ranked fourth in the list of mental illnesses, Obsessive-Compulsive Disorder (OCD) accounts for about ten percent of patients visiting medical centers. There have so far been various methods of treatment for OCD. An attempt was made in this study to determine the effectiveness of dialectical behavior therapy (DBT) on clinical signs and emotion regulation among patients with OCD. This was a quasi-experimental study involving intervention and control groups. The statistical population included all female patients referred to psychological counseling centers in Neyshabur during 2015. Each group comprised 15 patients selected through the convenient sampling method. The measurement tool was Yale-Brown's Obsessive Compulsive Scale ( $Y$ $B O C S)$ and Garnefski's Cognitive Emotion Regulation Questionnaire (CERQ). Training was provided for the intervention group during eight 90-minute sessions of dialectical behavior therapy, whereas the control group received no intervention. Analysis of covariance of the scores revealed that dialectical behavior therapy effectively reduced the observed symptoms and improved cognitive emotion regulation among patients. Generally, the results suggested that DBT alleviated OCD by modifying emotional responses of patients and that DBT could prove a highly effective treatment in this regard.

Keyword: dialectical behavior therapy, clinical symptoms, rumination, emotion regulation, obsessive-compulsive

\section{Introduction}

Obsessive-Compulsive Disorder (OCD) is characterized by specific uncontrollable, recurrent thoughts as well as impulsive, ritualized behaviors, where individuals feel as if their thoughts and actions were repeated in response to certain obsessive rules. The 12-month prevalence of OCD in the US is $1.2 \%$. Adult women are more likely to be affected than men are (American Psychiatric Association, 2015).

Throughout relevant research on OCD patients, defect observed in emotional regulation was reported as one of the major problems (Jacob et al.,2012; Allen et al., 2009; Fontenelle et al., 2012; Simon et al.,2014; Neumann et al.,2014). In recent years, increasing attention has been directed towards the role of emotional regulation and processing in a variety of disorders (Leahy et al.,2014). Emotion regulation disorder plays a significant role in borderline personality disorder (Schulze et al.,2015), anxiety disorder (Gong et al.,2015), self-esteem(Ritchie et al.,2015), eating disorders (Innamorati et al.,2015; Crockett et al.,2015), body-focused repetitive behaviors such as nail-biting and skin-picking (Roberts et al.,2013) and hoarding disorder (Fernández et al.,2013). There is a relationship between cognition and emotion, where emotional regulation contributes to depression, post-traumatic stress disorder and OCD (Taylor et al., 2007). Theoretically, rejection of cognitive therapy in treating anxiety disorders is partly associated with emotional avoidance and fear of anxiety caused by dysregulation (Leahy, 2007). When emotion is triggered to a troublesome degree of severity, it may be difficult to adopt other traditional cognitive-behavioral techniques, therefore, stress level can be escalated. Moreover, employment of behavioral techniques, particularly exposure techniques, may be difficult since they tend to aggravate emotional distress (Leahy et al.,2014). 
Dialectical Behavior Therapy (DBT) is a type of cognitive-behavioral therapy developed by Marsha Linehan for treating borderline personality disorder. DBT emphasizes on continuous assessment and collection of data associated with current behaviors, clear-cut definition of therapeutic goals, and collaborative relationship between therapist and client. Many components of dialectical behavior therapy such as problem-solving, exposure, skills training, contingency management, and cognitive modification have been prevalent for several years in cognitive and behavioral therapy. This method of treatment is very effective for patients who seek to control their overwhelming emotions(Linehan, 2013). Although cognitive behavioral therapy is an effective treatment for OCD, dialectical behavior therapy can help curtail emotional intensity and maintain emotional balance in times of crisis through training four basic skills, namely, mindfulness skills, interpersonal efficiency skills, emotion regulation, and distress tolerance skills (Mackay et al.,2012), taking a step beyond conventional cognitive-behavioral therapy. The third-wave treatments include those that affect coping strategies for negative emotions (Leahy et al.,2014). Mindfulness is an emotion-focused therapy effective in OCD (Strauss et al.,2015; Külz et al.,2014; Alizadeh et al.,2014; deZoysa, 2013; Hertenstein et al.,2012; Hanstede et al.,2008; Fairfax, 2008). Covering dialectical behavior therapy, the third-wave behavioral treatments can enhance flexible and adaptable ways of how individuals respond (Izadi et al.,2013). According to researchers, DBT is an effective treatment for emotion regulation (Neacsiu et al., 2014 ; Rizvi et al., 2014 ;Geddes et al., 2013; Jamilian et al., 2014; Linehan et al., 2015). Furthermore, the obtained results indicated that patients with OCD performed far more poorly in terms of emotional recognition and inhibition than the normal group since they had difficulty in processing and restraining emotional ambiguities (Ghamari-Givi et al., 2012). Dialectical behavior therapy was originally developed for patients with a history of attempting suicide. However, further research demonstrated that DBT entailed a hierarchical structure consisting of individual psychotherapy, group skills, training and counseling, which were also appropriate for treating other populations (Linehan et al., 2015).

Given its high prevalence, severity and diversity, OCD has been linked to numerous key functional difficulties among patients including job loss, marriage breakdown, and poor interpersonal relationships (Astekty et al., 2010). These indicated that more in-depth and updated research had to be conducted on OCD.

Results seem promising despite the fact that there is limited empirical evidence for DBT, especially as a treatment for OCD. To this end, this study attempted to determine the effectiveness of dialectical behavior therapy (DBT) on clinical signs and emotion regulation among patients with obsessive-compulsive disorder.

\section{Methodology}

This was a semi-experimental study involving pretest-posttest and control groups. The statistical population included all the female patients referred to medical centers providing psychological counseling services in Neyshabur during 2015. The sample population consisted of 30 participants selected through convenience sampling and randomly assigned to two experimental and control groups.

In the next stage, 30 patients with obsessive-compulsive disorder who received an OCD diagnostic interview based on DSM-V were placed into two groups, the treatment group $(n=15)$ and the waiting-list $(n=15)$. The inclusion criteria were: expert diagnosis of OCD based on DSM-V, patient's age being between 20 and 45 years, and minimum secondary-school education for the patients. The exclusion criteria were history of psychological treatment or medication for mental health problems within the previous six months and history of physical illnesses and drug abuse. The intervention group received training courses on dialectical behavior therapy for 8 weekly sessions, each lasting 90 minutes. The training stages of dialectical behavior therapy were applied based on the instructions prescribed by relevant literature (Linehan, 2013) as follow:

First session: Familiarization of participants with structural aspects of treatment, such as format, rules, duration of each session and therapeutic goals.

Second session: What-based mindfulness skills, serving the purpose of teaching lifestyle based on partnership combined awareness.

Third session: How-based mindfulness skills, aiming to raise awareness of real-time activities and taking a nonjudgmental stance. of reality.

Fourth session: Distress tolerance skills training, dedicated to survival strategies in times of crisis and recognition

Fifth Session: Emotion regulation training, including identification of emotions, reduction of vulnerability to negative emotions, and enhancing positive emotional events.

Sixth session: Learning mindfulness toward emotions, and resisting against intense emotional desires.

Seventh session: Learning interpersonal skills. 
Eighth session: Discussing the expansion of skills to real-life situations, and summing up the entire course.

Generally, based on the objectives of each session, the focus of attention would be on identification of situations, emotions, beliefs, and consequences leading to formation of behavior and inhibition. Moreover, each session involved practicing the newly learned skills and checking the preset assignments.

During this period, the waiting group received no psychological treatment. Before and after application of the independent variable, all subjects in both the intervention and waiting-list groups were assessed through Yale-Brown's Obsessive Compulsive Scale (Y-BOCS) and Garnefski's Cognitive Emotion Regulation Questionnaire (CERQ).

\subsection{Yale-Brown's Obsessive Compulsive Scale (Y-BOCS)}

This scale is a semi-structured interview assessing the severity of OCD symptoms in the current status of the disease. In fact, it assesses the time spent, interference, discomfort, resistance and self-control. Dadfar et al. reported that reliability of the scale was 0.98 , internal consistency coefficient was 0.89 , and retest reliability coefficient within two weeks was 0.84. Furthermore, its diagnostic validity through Beck's Depression Inventory and Hamilton's Anxiety Rating were calculated as 0.64 and 0.59 respectively (Izadi et al.,2014).

\subsection{Cognitive Emotion Regulation Questionnaire (CERQ).}

As a multidimensional, self-report questionnaire, CERQ was formulated by Garnefski et al. (2001) who reported ideal reliability and validity for this instrument. Moreover, CERQ contains 36 items on a five-point scale (from always or never), assessing a total of 9 cognitive coping strategies, including self-blame, other-blame, rumination, catastrophizing, positive refocus, refocus on planning, and positive reappraisal. The results of a study by Samani et al. suggested that e alpha coefficient for the subscales derived from CERQ had adequate internal consistency. The retest results within one-week interval for subscales ranged from 0.75 to 0.85 , indicating a desirable level of reliability. Furthermore, the correlation of subscales extracted from the questionnaire achieved a total score for DASS, suggesting ideal convergent and divergent validities(Samani et al., 2010).

Table 1: The average and standard deviation in the pre-test sample of variables

\section{Findings}

The mean and standard deviations for each variable in the pre-test and post-test in both experimental and control groups are shown in tables 1 and 2 respectively.

Table 1: Mean and standard deviation of sample subjects in a pretest involving the variables

\begin{tabular}{|c|c|c|c|c|c|c|c|}
\hline \multicolumn{2}{|r|}{ Variable } & Group & Minimum & Maximum & Mean & Standard Deviation & Number \\
\hline \multirow{6}{*}{ 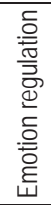 } & \multirow{2}{*}{ Positive emotion regulation } & treatment group & 40 & 68 & 51.80 & 9.02 & 15 \\
\hline & & control group & 44 & 72 & 53.20 & 8.22 & 15 \\
\hline & \multirow{2}{*}{ Negative emotion regulation } & treatment group & 56 & 79 & 67.20 & 9.29 & 15 \\
\hline & & control group & 55 & 75 & 62.46 & 5.96 & 15 \\
\hline & \multirow{2}{*}{ Emotion regulation } & treatment group & 99 & 156 & 119 & 16.04 & 15 \\
\hline & & control group & 100 & 135 & 115.66 & 9.47 & 15 \\
\hline \multirow{2}{*}{\multicolumn{2}{|c|}{ Clinical signs of OCD }} & treatment group & 14 & 30 & 24.20 & 4.21 & 15 \\
\hline & & control group & 11 & 23 & 24.53 & 4.71 & 15 \\
\hline
\end{tabular}

Similarly, the mean and standard deviation of subjects in the pre-test sample for each variable can be seen in the following table: 
Table 2: Mean and standard deviation of sample subjects in posttest concerning the variables

\begin{tabular}{|c|c|c|c|c|c|c|c|}
\hline \multicolumn{2}{|r|}{ Variable } & Group & Minimum & Maximum & Mean & Standard Deviation & Number \\
\hline \multirow{6}{*}{ 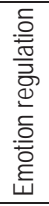 } & \multirow{2}{*}{ Positive emotion regulation } & treatment group & 50 & 88 & 63.4 & 10.08 & 15 \\
\hline & & control group & 40 & 60 & 52.26 & 8.30 & 15 \\
\hline & \multirow{2}{*}{ Negative emotion regulation } & treatment group & 60 & 88 & 71.60 & 7.48 & 15 \\
\hline & & control group & 50 & 79 & 60.40 & 7.18 & 15 \\
\hline & \multirow{2}{*}{ Emotion regulation } & treatment group & 117 & 163 & 125 & 14.78 & 15 \\
\hline & & control group & 93 & 136 & 112.66 & 11.83 & 15 \\
\hline \multirow{2}{*}{\multicolumn{2}{|c|}{ Clinical signs of OCD }} & treatment group & 12 & 30 & 18.40 & 3.96 & 15 \\
\hline & & control group & 12 & 31 & 24.46 & 4.98 & 15 \\
\hline
\end{tabular}

The results of statistical hypotheses indicated that equality of variances through Levene's test and normality were true $(\mathrm{p}<0.05)$.

The first hypothesis suggested that dialectical behavior therapy was effective on emotion regulation in patients with obsessive-compulsive disorder. Table 3 illustrates the results of analysis of covariance for testing the above hypothesis. The testing results were as follows:

Table 3: Summarizes the results of analysis of covariance concerning the mean scores of emotion regulation

\begin{tabular}{|l|c|c|c|c|c|c|}
\hline source & Sum of Squares & df & Mean Square & F & Sig & Partial Eta Squared \\
\hline Corrected Model & $6744 / 657^{a}$ & 2 & $3372 / 329$ & $45 / 131$ & $0 / 000$ & $0 / 770$ \\
\hline Pretest & $3003 / 824$ & 1 & $3003 / 824$ & $40 / 200$ & $0 / 000$ & $0 / 598$ \\
\hline grop & $2865 / 431$ & 1 & $2865 / 431$ & $38 / 348$ & $0 / 000$ & $0 / 587$ \\
\hline Error & $2017 / 510$ & 27 & $74 / 723$ & & & \\
\hline Total & $4688 / 3.000$ & 30 & & & & \\
\hline Corrected Total & $8762 / 167$ & 29 & & & & \\
\hline
\end{tabular}

Based on the data listed in Table 3, the results of analysis of covariance indicated there was a significant difference between the adjusted mean values of emotion regulation in patients with obsessive-compulsive disorder in intervention and control groups $(p<0.05)$. This implied that the hypothesis was proved, i.e. dialectical behavior therapy can be effective on emotion regulation of patients with obsessive-compulsive disorder. According to the mean values listed in Table 2, the mean emotion regulation of patients with OCD substantially increased after dialectical behavior therapy. The Eta Chi showed that nearly $59 \%$ of the variance for emotion regulation was explained for this variable.

The second hypothesis suggested that dialectical behavior therapy was effective on clinical signs in patients with obsessive-compulsive disorder. Table 4 illustrates the results of analysis of covariance for testing the above hypothesis. The testing results were as follows:

Table 4: Summarizes the results of analysis of covariance concerning the mean scores of clinical signs

\begin{tabular}{|l|c|c|c|c|c|c|}
\hline Source & Sum of Squares & df & Mean Square & F & Sig & Partial Eta Squared \\
\hline Corrected Model & $631 / 262^{a}$ & 2 & $315 / 631$ & $40 / 178$ & $0 / 000$ & $0 / 749$ \\
\hline Pretest & $355 / 229$ & 1 & $355 / 229$ & $45 / 219$ & $0 / 000$ & $0 / 626$ \\
\hline grop & $252 / 031$ & 1 & $252 / 031$ & $32 / 082$ & $0 / 000$ & $0 / 543$ \\
\hline Error & $212 / 105$ & 27 & $7 / 856$ & & & \\
\hline Total & $14625 / 000$ & 30 & & & & \\
\hline Corrected Total & $843 / 367$ & 29 & & & & \\
\hline
\end{tabular}

Based on the data listed in Table 3, the results of analysis of covariance indicated there was a significant difference between the adjusted mean values of clinical signs in patients with obsessive-compulsive disorder in intervention and control groups $(p<0.05)$. This implied that the hypothesis was proved, i.e. dialectical behavior therapy can be effective on clinical signs of patients with obsessive-compulsive disorder. According to the mean values listed in Table 2, the mean clinical signs of patients with OCD substantially decreased after dialectical behavior therapy. The Eta Chi showed that nearly $54 \%$ of the variance for clinical signs was explained for this variable. 


\section{Discussion}

This study attempted to determine the effectiveness of dialectical behavior therapy (DBT) on clinical signs and emotion regulation among patients with obsessive-compulsive disorder. The results obtained from adoption of DBT for women suffering from obsessive-compulsive disorder demonstrated its positive effect on curtailing the disorder. This was probably due to emphasis placed by DBT on training four areas of basic skills, namely, mindfulness, interpersonal efficiency skills, emotion regulation skills, and distress tolerance. Moreover, it could be associated with a great concentration on practical exercises and group work, thus building effective interpersonal communication. Another finding of this study highlighted the efficacy of DBT in emotion regulation, which was consistent with results obtained by Narimani et al. (2015), Zamani et al. (2014), Shamlu et al. (2014)who revealed that weekly training of DBT skills can enhance emotion regulation. Furthermore, these findings were consistent with those obtained (Neacsiu et al., 2014 ; Rizvi et al., 2014 ;Geddes et al., 2013; Jamilian et al., 2014; Linehan et al., 2015).

Patients with $O C D$ tend to deny or suppress their emotions, generally feeling terrible about emotions. In certain events, they find it difficult to control emotions. Hence, adoption of DBT techniques for emotion regulation can be helpful, including relaxation exercises, specific modes of attention, distraction, and many other emotion regulation strategies which can bring about appropriate emotion management. Previous studies have suggested that emotion regulation greatly contributes to repeated body-focused behavior such as hair-pulling, nail-biting, skin-picking (Roberts et al., 2013) and hoarding disorder (Fernández et al.,2013). Moreover, there is a relationship between avoidance of experience, emotion regulation, and obsessive-compulsive disorder (Fernández et al.,2013). Zahireddin et al., (2014) recommended that patients with OCD be provided with emotion-focused interventions and emotion regulation strategies .

According to Hashemi et al., ( 2014), patients with OCD tend to be substantially different in terms of how they employ emotion regulation strategies. Moreover, these strategies often appear as causal factors exacerbating the symptoms of OCD. The results of a study by Hashemi et al. indicated that cognitive emotion regulation strategies could provide a prediction model for OCD owing to their neuroticism and extroversion qualities. In this regard, Narimani et al. (2015) found that the mean scores of OCD patients for emotion regulation were lower than those achieved by normal individuals.

Emotion-focused therapy can provide an effective treatment for OCD. For instance, mindfulness is a key component of DBT (Strauss et al.,2015; Külz et al.,2014; Alizadeh et al.,2014; deZoysa, 2013; Hertenstein et al.,2012; Hanstede et al.,2008; Fairfax, 2008).. DBT comprises dialectical principles and techniques such as self-observation, which can lead to stability. Throughout DBT, mindfulness and behavioral practices are incorporated in a way that patients would be enabled to objectively observe their actions as well as their physiological, psychological, behavioral, and emotional consequences thereof. Recent research on DBT has yielded satisfactory results and sufficient rationale for the adoption of DBT in clinical procedures (Linehan et al., 2013). The more individuals become aware of their emotions, the lower the risk of avoidance shall be, thus the patient is enabled to deal with threatening situations (Rahimian Boogar et al., 2013). Moreover, DBT adopts a unique approach to emotions by focusing on both behavioral modification and reversing. Additionally, DBT is preferred over mindfulness owing to its concentration on behavioral modification exercises. One of the rarely-explored factors involved in the overall schema of cognitive-behavioral therapy is the individual's emotions and temperament since patients with OCD tend to have an extremely sensitive temperament. In that light, DBT strives to train individuals based on their specific temperaments. There were a few limitations in this study, including nonadministration of a follow-up test for evaluation of long-term effects, and non-consideration of social and cultural factors.

\section{Conclusions}

Generally, the results suggested that DBT can curtail OCD and be applied to modify emotional responses of patients as an effective, promising treatment.

\section{Acknowledgments}

We would like to express our gratitude toward all the participants in this research, as well as our colleagues at Nedaye Zendegi Counseling Center for cooperation in arrangement of training sessions.

\section{References}

American Psychiatric Association. (2015). Diagnostic and Statistical Manual of Mental Disorders(fifth edition). Seyed Mohammadi Y. 
Tehran: Ravan.

Alizadeh, A., \& Mohammadi, A. (2014). The effectiveness of mindfulness based exposure Therapy in Obsessive - Compulsive Disorder. J Res Behave Sci, 12(3), 342-50.

Allen, LB., \& Barlow, DH. (2009). Relationship of exposure to clinically irrelevant emotion cues and obsessive-compulsive symptoms.BehavModif, 33(6), 743-62.

Astekty, G., \& Pagett, T. (2010).Obsessive-compulsive disorder. Ghasemzadeh HL. Tehran: Arjmand.

Crockett, AC., Myhre, SK., \& Rokke, PD. (2015). Boredom proneness and emotionregulation predict emotional eating.J Health Psychol, 20(5), 670-80.

DeZoysa, P. (2013).The use of mindfulness practice in the treatment of a case of obsessive compulsive disorder in Sri Lanka. J Relig Health, 52(1), 299-306.

Fairfax, H . (2008). The use of mindfulness in obsessive compulsive disorder: suggestions for its application and integration in existing treatment.Clin Psychol Psychother, 15(1),53-9.

Fernández de la Cruz, L., Landau, D., lervolino, AC., Santo, S., Pertusa, A., Singh, S. (2013).Experiential avoidance and emotionregulation difficulties in hoarding disorder.J Anxiety Disord, 27(2), 204-9.

Fontenelle, LF., Harrison, BJ., Pujol, J., Davey, CG., Fornito, A., \& Bora, E. (2012). Brain functional connectivity during induced sadness in patients with obsessive-compulsive disorder. J Psychiatry Neurosci, 37(4), 231-40.

Geddes, K., Dziurawiec, S., \& Lee, CW.( 2013).Dialectical Behaviour Therapy for the Treatment of Emotion Dysregulation and Trauma Symptoms in Self-Injurious and Suicidal Adolescent Females: A Pilot Programme within a Community-Based Child and Adolescent Mental Health Service.Psychiatry J, 145219.

Ghamari-Givi, H., Imani, H., Barahmand, O., \& Sadeghi-Movahed F. (2012). The Investigation of Emotional Inhibition and Recognition in Patients with Obsessive-Compulsive. Journal of Clinical Psychology, 2 (14), 9-18.

Gong, L., Li, W., Zhang, D., \& Rost, DH. (2015). Effect of emotionregulation strategies on anxiety during job interview in chinese college students.Anxiety Stress Coping, 1-17.

Hanstede, M., Gidron, Y., \& Nyklícek, I. (2008). The effects of a mindfulness intervention on obsessive-compulsive symptoms in a nonclinical student population. J NervMent Dis , 196(10), 776-9.

Hashemi, T., Mahmoud alilou, M., \& Hosseinzadeh, S. (2014). Structural relationships of cognitive emotion regulation, extraversion and neuroticism with Obsessive- Compulsive symptoms in non-clinical people. Journal of Behavioral Sciences, 8(4),365-73.

Hertenstein, E., Rose, N., Voderholzer, U., Heidenreich, T., Nissen, C., Thiel, N., et al. (2012). Mindfulness-based cognitive therapy in obsessive-compulsive disorder -A qualitative study on patients'experiences. BMC Psychiatry, 12, 185.

Innamorati, M., Imperatori, C., Harnic, D., Erbuto, D., Patitucci, E., Janir, L., et al. (2015). Emotion Regulation and Mentalization in People at Risk for Food Addiction. Behav Med, 0 .

Izadi, R., Neshatdust, HT., Asgari, K., \& Abedi, MR. (2014). Comparison of the efficacy of Acceptance and Commitment Therapy and Cognitive-Behavior Therapy on symptoms of treatment of patients with obsessive- compulsive disorder. J Res Behave Sci, 12(1), 19-33.

Izadi, R., \& Abedi, MR. (2013). Alleviation of obsessive symptoms in treatment-resistant obsessive-compulsive disorder using acceptance and commitment-based therapy, 17 (3), 86-275.

Jacob, ML., Morelen, D., Suveg C., Brown Jacobsen, AM., \& Whiteside, SP. (2012).Emotional, behavioral, and cognitive factors that differentiate obsessive-compulsive disorder and other anxiety disorders in youth.Anxiety Stress Coping, 25(2), 229-37.

Jamilian, HR., Malekirad, AA., Farhadi, M., Habibi, M., \& Zamani, N. (2014). Effectiveness of group dialectical behavior therapy (based on core distress tolerance and emotion regulation components) one expulsive anger and impulsive behaviors. Glob $J$ Health Sci,6(7),116-23.

Külz, AK., \& Rose, N. (2014).Mindfulness based cognitive therapy (MBCT) in patients with obsessive-compulsive disorder--an adaptation of the original program. Psychother Psychosom Med Psychol, 64(1), 35-40.

Leahy, R.L., Tirch, D., \& Napolitano, LA. (2014). Emotion regulation in psychotherapy. Mansouri Rad A. Tehran: Arjmand.

Leahy, R. L. (2007). Emotional Schemas and Self-Help: Homework Compliance and Obsessive-Compulsive Disorder Cognitive and Behavioral Practice, 14( 3): 297-302 .

Linehan, MM., \& Wilks, CR. (2015).The Course and Evolution of Dialectical Behavior Therapy.Am J Psychother, 69(2), 97-110.

Linehan, MM. (2013). Guide to run Dialectical Behavior Therapy for Borderline Personality Disorder.Alavi Kh. Tehran: Arjmand.

MacKay, M., Bertenly, J. (2012).Dialectical behavior therapy techniques. Hamidpour H,Jomeh Pour H,Andruze Z. Tehran: Arjmand.

Narimani, M., Bagiyan-Kulemarez, MJ., Bavand-Poouri, AR., \& Bakhti, M. (2015).The Effectiveness of Group Psychotherapy based on Dialectical Behavior Therapy (DBT) on the Degree of Craving and Difficulty in Emotional Self-regulation of Methamphetamine Users. Journal of Clinical Psychology, 4 (24).

Narimani, M., Almardani-Some'eh, S., Mikaeeli, N., \& Basharpoor, S. (2015). The Comparison of Intolerance of Uncertainty, Emotion Regulation and Marital Satisfaction in OCD-Patients and Normal Individuals. Journal of Clinical Psychology, 4 (24).

Neacsiu, AD., Eberle, JW., Kramer, R., Wiesmann, T., \& Linehan, MM. (2014).Dialectical behavior therapy skills for transdiagnostic emotion dysregulation: a pilot randomized controlled trial.Behav Res Ther,59,40-51.

Neumann, WJ., Huebl, J., Brücke, C., Gabriëls, L., Bajbouj, M., Merkl, A. , et al. (2014). Different patterns of local field potentials from limbic DBS targets in patients with major depressive and obsessive compulsive disorder.Mol Psychiatry, 19(11), 1186-92.

Rahimian Boogar, I., Kianersi, F., \& Tarbaran, F. (2013). Investigating the relationships of emotional schema, cognitive-behavioral avoidance and cognitive-affective system with generalized anxiety disorder and obsessive-compulsive disorder. 
Pejouhandeh., 18(5),267-276.

Rizvi, SL., \& Steffel, LM. (2014). A pilot study of 2 brief forms of dialectical behavior therapy skills training for emotion dysregulation in college students. J Am Coll Health, 62(6),434-9.

Ritchie, TD., Sedikides, C., \& Skowronski, JJ. (2015). Emotions experienced at event recall and the self: Implications for the regulation of self-esteem, self-continuity and meaningfulness.Memory, 1-15.

Roberts, S., O'Connor, K., \& Bélanger, C. (2013). Emotionregulation and other psychological models for body-focused repetitive behaviors.ClinPsychol Rev, 33(6), 745-62.

Samani, S., \& Sadeghi, L. (2010).Psychometric Properties of the Cognitive Emotion Regulation Questionnaire. Journal of Psychological Models and Methods, 1(1), 51-62.

Schulze, L., Schmahl, C., \& Niedtfeld, I. (2015).Neural Correlates of Disturbed Emotion Processing in Borderline Personality Disorder: A Multimodal Meta-Analysis. Biol Psychiatry. pii: S0006-3223(15)00278-4.

Shamloo, N. Modarres Gharavi, M., \& Asgharipour, N.(2014). The effectiveness of dialectical behavior therapy based on emotion regulation training on depression symptoms. Journal of Fundamentals of Mental Health,16(1), 102-108.

Simon, D., Adler, N., Kaufmann, C., \& Kathmann, N. (2014). Amygdala hyperactivation during symptom provocation in obsessivecompulsive disorder and its modulation by distraction. NeuroimageClin, 26(4), 549-57.

Strauss, C., Rosten, C., Hayward, M., Lea, L., Forrester, E., Jones, AM. (2015). Mindfulness-based exposure and response prevention for obsessive compulsive disorder: study protocol for a pilot randomized controlled trial. Trials, 16,167.

Taylor, SF., \& Liberzon, I. (2007). Neural correlates of emotionregulation in psychopathology. Trends Cogn Sci, 11(10), 413-8.

Zahiroddin, A., Agahi, Z., Borjali, A., \& Rajezi, S. (2014). Comparison of emotion regulation strategies among individuals with obsessivecompulsive disorder and substance abusers. Pejouhandeh,19(1),18-24.

Zamani, N., Farhadi, M., Jamilian, HR., \& Habibi, M. (2014). Effectiveness of group dialectical behavior therapy (based on core distress tolerance and emotion regulation components) on Expulsive Anger and Impulsive Behaviors. Arak Medical University Journal (AMUJ), 17(92), 53-61. 